\title{
Perioperative seizure in patients with glioma is associated with longer hospitalization, higher readmission, and decreased overall survival
}

\author{
Michael C. Dewan, MD, Gabrielle A. White-Dzuro, BA, Philip R. Brinson, MPH, \\ Reid C. Thompson, MD, and Lola B. Chambless, MD \\ Department of Neurological Surgery, Vanderbilt University School of Medicine, Nashville, Tennessee
}

\begin{abstract}
OBJECTIVE Seizures are among the most common perioperative complications in patients undergoing craniotomy for brain tumor resection and have been associated with increased disease progression and decreased survival. Little evidence exists regarding the relationship between postoperative seizures and hospital quality measures, including length of stay (LOS), disposition, and readmission. The authors sought to address these questions by analyzing a glioma population over 15 years.

METHODS A retrospective cohort study was used to evaluate the outcomes of patients who experienced a postoperative seizure. Patients with glioma who underwent craniotomy for resection between 1998 and 2013 were enrolled in the institutional tumor registry. Basic data, including demographics and comorbidities, were recorded in addition to hospitalization details and complications. Seizures were diagnosed by clinical examination, observation, and electroencephalography. The Student t-test and chi-square test were used to analyze differences in the means between continuous and categorical variables, respectively. Multivariate logistic and linear regression was used to compare multiple clinical variables against hospital quality metrics and survival figures, respectively.
\end{abstract}

RESULTS In total, 342 patients with glioma underwent craniotomy for first-time resection. The mean age was 51.0 \pm 17.3 years, $192(56.1 \%)$ patients were male, and the median survival time for all grades was 15.4 months (range $6.2-24.0$ months). High-grade glioma (Grade III or IV) was seen in $71.9 \%$ of patients. Perioperative antiepileptic drugs were administered to $88 \%$ of patients. Eighteen (5.3\%) patients experienced a seizure within 14 days postoperatively, and $9(50 \%)$ of these patients experienced first-time seizures. The mean time to the first postoperative seizure was 4.3 days (range $0-13$ days). There was no significant association between tumor grade and the rate of perioperative seizure (Grade I, 0\%; II, 7.0\%; III, 6.1\%; IV, 5.2\%; $p=0.665$ ). A single ictal episode occurred in 11 patients, while 3 patients experienced 2 seizures and 4 patients developed 3 or more seizures. Compared with their seizure-free counterparts, patients who experienced a perioperative seizure had an increased average hospital (6.8 vs 3.6 days, $p=0.032$ ) and ICU LOS (5.4 vs 2.3 days; $p<0.041$ ). Seventy-five percent of seizure-free patients were discharged home in comparison with $55.6 \%$ of seizure patients $(p=0.068)$. Patients with a postoperative seizure were significantly more likely to visit the emergency department within 90 days (44.4\% vs $19.0 \%$; OR 3.41 [95\% $\mathrm{Cl} 1.29-9.02], \mathrm{p}=0.009$ ) and more likely to be readmitted within 90 days ( $50.0 \%$ vs $18.4 \%$; OR 4.45 [ $95 \% \mathrm{Cl} 1.69-11.70$ ], $p=0.001$ ). In addition, seizure-free patients had a longer median overall survival (15.6 months [interquartile range 6.6-24.4 months] vs 3.0 months [interquartile range $1.0-25.0$ months]; $p=0.013$ ).

CONCLUSIONS Patients with perioperative seizures following glioma resection required longer hospital and ICU LOS, were readmitted at higher rates than seizure-free patients, and experienced shorter overall survival. Biological and clinical factors that predispose to the development of seizures after glioma surgery portend a worse outcome. Efforts to identify these factors and reduce the risk of postoperative seizure should remain a priority among neurosurgical oncologists. http://thejns.org/doi/abs/10.3171/2015.10.JNS151956

KEY WORDS glioma; seizure; length of stay; readmission; survival; oncology

ABBREVIATIONS ACA = Affordable Care Act; $A E D$ = antiepileptic drug; ED = emergency department; EEG = electroencephalography; EOR = extent of resection; GTR = gross-total resection; IQR = interquartile range; KPS = Karnofsky Performance Scale; LOS = length of stay; NTR = near-total resection; STR = subtotal resection. SUBMITTED August 20, 2015. ACCEPTED October 2, 2015.

INCLUDE WHEN CITING Published online February 19, 2016; DOI: 10.3171/2015.10.JNS151956. 
$\mathrm{S}$ EIZURES are among the most common complications in patients with brain tumors, affecting between $20 \%$ and $40 \%$ of patients in most reports. ${ }^{8,12,15}$ While patients with brain tumors are at an increased risk of seizures, this risk is compounded in patients who undergo craniotomy for tumor resection. ${ }^{13,25,32}$ Postoperative seizures are particularly problematic as they can lead to hemorrhage, cerebral hypoxia, increased intracranial pressure, and neurological deterioration, ${ }^{23-25}$ all of which can result in delayed postoperative recovery and long-term morbidity. ${ }^{28}$ Perhaps most profoundly, seizures in brain tumor patients have been associated with increased disease progression and poorer overall survival. ${ }^{4,47}$ These reports outline the influence of postoperative seizures on traditional provider-based measures such as neurological outcome and survival. However, the relationship between perioperative seizures and hospital quality metrics, including length of stay (LOS), hospital disposition, and readmissions has not been described.

During a period of shrinking health care dollars and increased scrutiny of postoperative morbidity, the impact of perioperative seizures is becoming more important and more apparent. Since 2012, the Centers for Medicare and Medicaid Services has conferred penalties for readmissions related to common disease states such as pneumonia and heart failure. ${ }^{35,36}$ Indeed, the day when reimbursement decisions and health care quality ratings are based on the sequelae of events, such as postoperative seizure, appears to be on the horizon. The purpose of this study is 2-fold: 1) identify the incidence of and factors contributing to perioperative seizure in a glioma population; and 2) examine the relationship between seizures and common health care quality metrics. In quantifying this relationship, we highlight the magnitude of this morbid complication and emphasize the importance of seizure risk reduction.

\section{Methods \\ Patient Population}

Institutional review board approval was obtained for this study. Patients with glioma who underwent craniotomy for resection between 1999 and 2013 were enrolled in our institutional tumor registry after informed consent was obtained. Basic data, including demographics, presenting symptoms, as well as tumor grade and location, were recorded. Patients included in the analysis were those who underwent first-time cytoreductive surgery for pathologically confirmed glioma and underwent at least 12 months of follow-up or the date of death was known (if sooner than 12 months).

\section{Perioperative Antiepileptic Drug Administration}

Individual surgeon preferences dictated whether seizure-naive patients received prophylactic antiepileptic drugs (AEDs). Four surgeons treated this 342-patient population. Two of the surgeons always administer AED prophylaxis in this population, while another does not. The remaining surgeon administered prophylaxis for most of the study duration; however, more recently, he has changed his practice to withhold prophylaxis. Importantly, aside from a history of prior seizures, the decision to ad- minister or not administer prophylaxis was independent of patient-specific factors.

In general, seizure-naive patients who were prescribed prophylaxis received $500 \mathrm{mg}$ levetiracetam intravenously during induction and every 12 hours thereafter for 7 days. As soon as patients could tolerate oral medications, the oral form of levetiracetam was substituted. Patients previously on a regimen of AEDs received their home dose immediately preoperatively and, when indicated, received an additional dose intraoperatively.

\section{Outcomes Measurement}

In-hospital, postoperative, clinical seizure was diagnosed clinically at the bedside by the ICU and floor staff. When appropriate, electroencephalography (EEG) was used and neurology consultation was sought. If suspected seizure activity occurred without the benefit of direct medical observation, the summation of witness observation and/or patient examination was used to confirm or exclude seizure activity. Accordingly, witnessed generalized episodes depended on a reliable description of the event that was convincing of an ictal event to multiple team members. Simple partial seizures were often described by the patient. Generalized, unwitnessed seizures were the most problematic and were only considered seizure if tongue biting and urinary incontinence were observed during the patient encounter. To minimize data contamination and ensure a pure seizure cohort, patients with suspected but unconfirmed seizures were removed from all analyses that compared outcomes between the seizure and seizure-free cohorts.

At our institution, gross-total resection (GTR) is considered to have been achieved when both the neurosurgeon and radiologist characterize postoperative MRI (within 8-24 hours of surgery completion) as having no residual contrast enhancement (high-grade glioma) or no residual T2-hyperintesity (T2-predominant, low-grade lesion). A near-total resection (NTR) is one in which $95 \%$ to $99 \%$ of a preoperative contrast-enhancing or T2-hyperintense volume has been removed, or when the senior radiologist cannot rule out residual tumor. A subtotal resection (STR) is considered when $<95 \%$ of the tumor is removed.

Perioperative seizures were considered those that occurred between the time of surgery and 14 days postoperatively. Outcomes were compared after subdividing patients into 2 groups based on whether a perioperative seizure had occurred. The primary outcomes were LOS in the hospital and ICU, discharge disposition, 90-day emergency department (ED) visits and readmissions, and overall survival.

\section{Statistical Analysis and Data Storage}

Parametric data were given as mean \pm standard deviation and were compared using the Student t-test. Nonparametric data were given as median (interquartile range $[\mathrm{IQR}]$ ) and compared using the Mann-Whitney U-test. Shapiro-Wilk testing was used to determine the normality of the continuous variables. Dichotomous data were initially compared using the chi-square test, and comparisons of the binary variables with fields less than 5 frequencies were compared 
using the Fisher's exact test. Multivariate logistic regression was then applied to allow adjustment for potential confounding covariates. Given the interval nature of the survival figures, multivariate linear regression was used to compare overall survival differences. Kaplan-Meier analysis was conducted, and survival data were compared and reported using the Breslow (Generalized Wilcoxon) test. A p value $<0.05$ was considered statistically significant. With regard to readmission risk factors, variables with a $p$ value $<0.1$ on the univariate analysis were entered into a multivariate analysis (logistic regression). All statistical analyses were performed using IBM SPSS software (version 22). Study data were collected and managed using Research Electronic Data Capture (REDCap) tools hosted at Vanderbilt University Medical Center. ${ }^{17}$

\section{Results \\ Patient Population}

Between 1999 and 2013 at our institution, 342 patients with glioma underwent craniotomy for first-time supratentorial tumor resection and met the inclusion criteria. The mean age was $51.0 \pm 17.3$ years, and $192(56.1 \%)$ patients were male. The majority of patients harbored a Grade IV glioma (196 patients; 57.3\%), followed by Grade II (74 patients; $21.6 \%$ ) and Grade III (49 patients; 14.3\%) lesions. Grade I tumors were found in a small minority (20 patients; 5.8\%) of patients. Astrocytoma (255 patients; $74.6 \%$ ) was the most common cell type, followed by oligodendroglioma (52 patients; 15.2\%), ependymoma (19 patients; 5.6\%), and oligoastrocytoma (16 patients; $4.7 \%$ ).

\section{Clinical Presentation}

The most common presentation was seizure (37.7\%), followed by headache (21.0\%), personality/cognitive changes (14.9\%), and focal motor/sensory deficit (11.9\%). Seizures were diagnosed anytime preoperatively in $43.2 \%$ of patients. Preoperative imaging identified tumor involvement in the frontal, temporal, and parietal lobes in $35.4 \%$, $29.9 \%$, and $20.4 \%$ of patients, respectively. Occipital (4.4\%) and diencephalic (4.1\%) lesions were less common.

With regard to the preoperative clinical and tumor characteristics, patients who experienced a perioperative seizure did not differ significantly from those who remained seizure free (Table 1). The mean age of the seizure patients was 52.0 years in comparison with 50.9 years in the seizure-free group ( $\mathrm{p}=0.796)$. Mean Karnofsky Performance Scale (KPS) scores were also similar between respective groups when compared both as raw and categorized values $(\mathrm{p}=0.051$ and 0.524$)$. Tumor cell type and histological grade were also similar between both groups $(\mathrm{p}=0.0 .441$ and $\mathrm{p}=0.665)($ Table 1$)$.

\section{Perioperative Seizures and AED Administration}

The vast majority of patients $(88.0 \%)$ received AED postoperatively. Levetiracetam was the drug of choice for $73.7 \%$ of patients, followed by phenytoin and oxcarbazepine (24\% and $1.7 \%$, respectively). Among the patients who were given routine seizure prophylaxis, the mean and median treatment durations were 9.2 and 7.0 days, respectively.
Eighteen patients $(5.3 \%)$ experienced a seizure within 14 days of surgery, $9(50 \%)$ of whom experienced firsttime seizures (Table 2). The mean and median times to first postoperative seizure were 4.3 and 3 days, respectively (range $0-13$ days) (Table 2 ). The majority (11 patients; $61.1 \%$ ) experienced only a single seizure, while 7 patients (38.9\%) experienced 2 or more seizures and 1 patient suffered an episode of status epilepticus. Eight seizures were generalized tonic-clonic, 5 seizures were simple partial, and the remaining were either complex partial or unspecified. Two patients underwent re-resection within 90 days of index surgery; neither experienced an early or late seizure. In 7 of the 342 patients (2\%), a perioperative seizure was suspected based on a patient or witness description but could not be reliably confirmed. In an effort to eliminate ambiguity, these 7 patients were not included in the analyses comparing differences between seizure and seizure-free patients.

Among the 18 patients who experienced confirmed perioperative seizures, a total of 32 events were diagnosed. Twenty-three seizures (72\%) occurred within the first 7 days (i.e., the median perioperative AED treatment window), while an additional 9 (28\%) seizures occurred between 8 and 13 days postoperatively. Late seizures (those defined as occurring between 15 and 90 days after index surgery) were identified in 22 patients. Including the 18 patients who developed a perioperative seizure, a total of 25 patients had a seizure within 1 month. At the 2- and 3-month intervals, an additional 8 and 7 patients developed a seizure, respectively, bringing the total number of patients with 90-day seizures to 40 (11.7\%) patients. The median time to first late seizure was 50 days (range 18-88 days).

The existence of preoperative seizures did not predict the rate of observed perioperative seizures. Among the 18 patients who developed a perioperative seizure, half had a prior history of seizures, while half experienced a first-time seizure perioperatively $(p=0.537)$. Similarly, the administration of perioperative prophylaxis did not influence the rate of seizures in patients without a history of seizures. Among 191 seizure-naive patients receiving prophylaxis, $8(4.2 \%)$ patients had a seizure within 14 days in comparison with 1 of $38(2.6 \%)$ patients who were not on prophylaxis $(\mathrm{p}=0.39)$. Achieving GTR was associated with a decreased incidence of perioperative seizure $(\mathrm{p}=$ 0.022 ). The seizure-protective advantage of GTR was lost when these patients were clustered with those who underwent NTR, as compared with patients who underwent $\operatorname{STR}(\mathrm{p}=0.103)$.

\section{Quality Metrics and Health Care Burden}

Compared with their seizure-free counterparts, patients experiencing a perioperative seizure had increased average hospital (6.8 vs 3.6 days; $p=0.032$ ) and ICU (5.4 vs 2.3 days; $\mathrm{p}=0.041)$ LOS (Table 1). No modifiable factors that were measured, including extent of resection (EOR), postoperative AED administration, and discharge disposition, influenced LOS durations. Seventy-five percent of patients who remained seizure free in the hospital were discharged home, while only $55.6 \%$ with a seizure were discharged home. This difference did not reach statistical significance 
TABLE 1. Patient and tumor characteristics stratified by perioperative seizure occurrence for 335 patients with glioma*

\begin{tabular}{|c|c|c|c|}
\hline Variable & Perioperative Seizure $(n=18)$ & No Perioperative Seizure $(n=317)$ & p Value \\
\hline Mean age $\pm S D$ in yrs & $52.0 \pm 15.3$ & $50.9 \pm 17.5$ & 0.796 \\
\hline Male sex & $14(77.8)$ & $173(54.6)$ & 0.054 \\
\hline Median KPS score [IQR] & $70[60-70]$ & $80[60-90]$ & 0.051 \\
\hline KPS score $\geq 70$ & $12(66.7)$ & $230(72.5)$ & 0.524 \\
\hline Temporal location & $4(22.2)$ & $90(28.3)$ & 0.814 \\
\hline Histology & & & 0.441 \\
\hline Astrocytoma & $12(66.7)$ & $239(75.2)$ & \\
\hline Oligodendroglia & $4(22.2)$ & $46(14.5)$ & \\
\hline Oligoastrocytoma & $0(0.0)$ & $15(4.7)$ & \\
\hline Ependymoma & $2(11.1)$ & $17(5.3)$ & \\
\hline Tumor grade & & & 0.665 \\
\hline I & $0(0.0)$ & $20(6.4)$ & \\
\hline II & $5(27.8)$ & $66(21.0)$ & \\
\hline III & $3(16.7)$ & $46(14.6)$ & \\
\hline IV & $10(55.6)$ & $182(58.0)$ & \\
\hline \multicolumn{4}{|l|}{ EOR } \\
\hline STR & $10(55.6)$ & $113(35.5)$ & 0.103 \\
\hline NTR & $7(38.9)$ & $108(34.0)$ & 0.484 \\
\hline GTR & $1(5.6)$ & $96(29.3)$ & 0.022 \\
\hline Preoperative seizure & $9(50.0)$ & $135(42.6)$ & 0.537 \\
\hline Postoperative AED & $17(94.4)$ & $278(87.7)$ & 0.39 \\
\hline Mean hospital LOS in days & $6.8 \pm 2.1$ & $3.6 \pm 1.6$ & 0.032 \\
\hline Mean ICU LOS in days & $5.4 \pm 2.3$ & $2.3 \pm 1.7$ & 0.041 \\
\hline Discharge to home & $10(55.6)$ & $234(75.0)$ & 0.068 \\
\hline 90-day ED visit & $8(44.4)$ & $60(19.0)$ & 0.009 \\
\hline 90-day readmission & $9(50.0)$ & $58(18.4)$ & 0.001 \\
\hline Median survival in mos [IQR] & $3.0[1.0-25.0]$ & $15.6[6.6-24.4]$ & 0.037 \\
\hline
\end{tabular}

$(\mathrm{p}=0.068)$. Forty-four percent of seizure patients visited the ED within 90 days relative to $19 \%$ without a perioperative seizure $(\mathrm{p}=0.009)$. Likewise, $50 \%$ of seizure patients required readmission within 90 days relative to $18 \%$ in the seizure-free cohort ( $\mathrm{p}=0.001)$ (Fig. 1). Applying logistic regression, patients in the seizure cohort were more likely to visit the ED within 90 days of discharge (OR 3.41 [95\% CI 1.29-9.02], $p=0.009$ ) and were more likely to be readmitted within 90 days (OR 4.45 [95\% CI 1.69-11.70], $\mathrm{p}=0.001$ ) (Table 3). Regardless of seizure status, those discharged to home, rather than to a rehabilitation facility or nursing home, were less likely to require readmission (OR 0.54, $\mathrm{p}=0.036$ ). Readmission rates, however, were not influenced significantly by the preoperative KPS score, tumor grade, EOR, AED administration, or radiation treatment (Table 3). The most common reasons for readmission among all patients were altered mental status or new neurological deficits (23.8\%), infectious complications (16.3\%), seizures (11.3\%), and chemotherapy-related morbidity (7.5\%). Among the 9 patients in the seizure cohort who were readmitted within 90 days, 3 (33\%) patients were readmitted for seizures.

Perioperative seizures also resulted in additional health care resource utilization. Thirteen (72.2\%) patients who experienced a seizure underwent EEG monitoring and 9 $(50 \%)$ received a formal neurology consultation (Table 2). An in-patient neurology consultation was accompanied by an outpatient neurology clinic visit at a time considered appropriate by the consulting neurologist. Imaging (e.g., CT) in the wake of an ictal episode was obtained in 15 $(83.3 \%)$ patients. Additionally, patients in the seizure cohort spent on average more than 3 additional days in the hospital $(\mathrm{p}=0.032)($ Table 1$)$.

\section{Overall Survival}

Among all patients (all histological grades included), the mean and median survival periods were $21.7 \pm 26.2$ and 15.4 months (IQR 6.17-23.7 months), respectively. The median overall survival was significantly lower for patients who had a perioperative seizure ( 89 vs 468 days, or 3.0 vs 15.6 months; $p=0.013$ ) (Fig. 2). The difference in mean survival was less pronounced but similarly significant $(405 \pm 58$ vs $670.2 \pm 141$ days, or 13.5 vs 22.3 months; $\mathrm{p}=0.025)$, although because the survival frequencies were nonnormally distributed, nonparametric reporting is favored. Overall survival differences in relation to seizure 
TABLE 2. Characteristics of 18 patients who experienced perioperative seizure

\begin{tabular}{|c|c|}
\hline Variable & Value (\%) \\
\hline Preoperative seizure(s) & $9(50 \%)$ \\
\hline \multicolumn{2}{|l|}{ Tumor grade } \\
\hline II & $5(27.8)$ \\
\hline III & $3(16.7)$ \\
\hline IV & $10(55.6)$ \\
\hline \multicolumn{2}{|l|}{ Tumor location } \\
\hline Frontal & $6(33.3)$ \\
\hline Temporal & $4(22.2)$ \\
\hline Parietal & $3(16.7)$ \\
\hline Other* & $5(27.8)$ \\
\hline \multicolumn{2}{|l|}{ Extent of resection } \\
\hline STR & $10(55.6)$ \\
\hline NTR & $7(38.9)$ \\
\hline GTR & $1(5.6)$ \\
\hline \multicolumn{2}{|l|}{ Time to first seizure, $P O D$} \\
\hline Mean (range) & $4.3(0-13)$ \\
\hline Median [IQR] & $3[0-6.5]$ \\
\hline \multicolumn{2}{|l|}{ No. of perioperative seizures } \\
\hline Mean (range) & $1.78(1-5)$ \\
\hline Median [IQR] & $1[1-2.5]$ \\
\hline Seizure-related imaging $\dagger$ & $15(83.3)$ \\
\hline Neurology consultation $†$ & $9(50)$ \\
\hline EEG obtained $†$ & $13(72.2)$ \\
\hline
\end{tabular}

$\mathrm{POD}=$ postoperative day.

* Includes occipital, periventricular, and deep locations.

$\dagger$ Includes test or service provided in inpatient or ED setting.

status remained significant after adjusting for tumor grade and EOR $(\mathrm{p}=0.026)$.

Poorly controlled seizures, which were present in 6 patients, were themselves not predictive of decreased overall survival. However, among the 18 patients with a perioperative seizure, 1 died of a seizure-related event. Following a seizure on Postoperative Day 7, she was intubated, became bradycardic, and died shortly thereafter. This patient had experienced another seizure earlier in the postoperative period (Postoperative Day 3) with an apneic spell, but she recovered uneventfully.

\section{Discussion}

Among the 342 patients who underwent craniotomy for glioma resection, $18(5.3 \%)$ patients developed a seizure within 14 days postoperatively. Relative to those who were seizure free, these patients remained in the hospital nearly twice as long, which included a significantly longer ICU stay. Patients in the seizure cohort were more than 3 times more likely than their seizure-free counterparts to visit the ED within 90 days of discharge and over 4 times more likely to be readmitted over the same period. Furthermore, median overall survival was significantly lower in patients who suffered a seizure within 14 days of tumor resection. To the best of our knowledge, this is the first report to di-

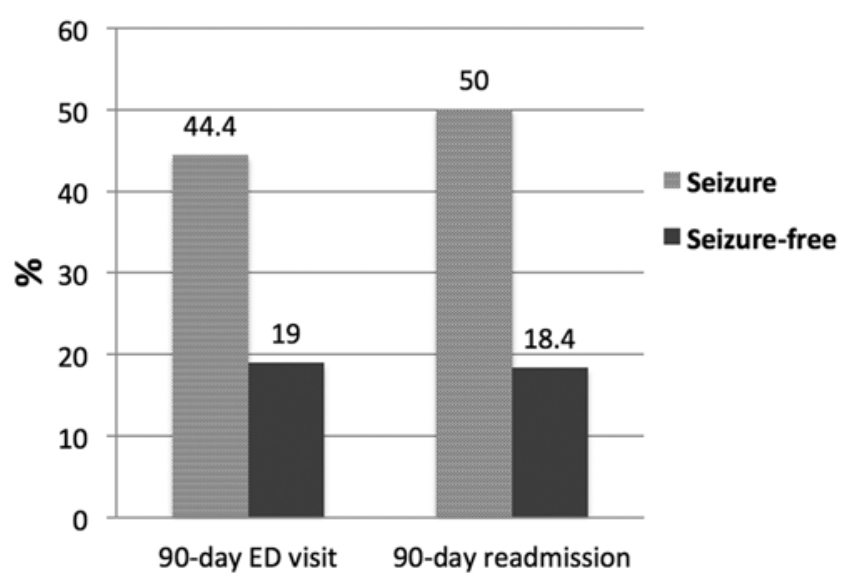

FIG. 1. Rates of 90-day ED visits and 90-day hospital readmissions among 18 seizure patients and 317 nonseizure patients. Both the ED visit $(p=0.009)$ and readmission $(p=0.001)$ rates were significantly higher in patients who experienced a perioperative seizure.

rectly link perioperative seizures after glioma resection to prolonged hospital stay, hospital readmission, and overall survival.

\section{Seizures and Health Care Quality Accountability}

At a time when health care costs are rising and increased pressure is placed on hospitals and physicians to provide quality care, postcraniotomy morbidity such as seizures must be closely examined. Several reports indicate that readmissions for preventable morbidity-particularly those related to infectious and thromboembolic disease-cost the health care system tens of thousands of dollars per patient. ${ }^{7,11,18,20}$ Among readmitted patients with intracranial tumors specifically, preventable complications are the culprit in nearly a third of cases. . $^{529,33}$

The magnitude of this problem was illustrated in the 2012 report commissioned by the Alliance for Home Health Quality and Innovation (AHHQI), which indicated that hospital readmissions more than doubled the cost of care delivered to patients treated over a spectrum of conditions. ${ }^{7}$ Two years earlier, the Affordable Care Act (ACA) was signed into law and called for improvement in the quality of health care delivery by making hospitals and providers accountable for avoidable costs. ${ }^{36}$ Section 3025 of the ACA outlined a revised payment plan that now penalizes providers for hospital readmissions..$^{35}$

Even prior to these measures, few would deny the importance of avoiding medical complications. And, while recent legislation may catalyze the discussion, efforts to reduce such complications have always been a primary driving force in medical research and health care delivery, the field of neurosurgery being no exception. Regarding neurosurgical oncology specifically, it is a common practice for surgeons to administer AEDs with the intent of seizure reduction. The majority of patients included in this study were placed on AED postoperatively, though even those who were not on prophylaxis did not experience a higher rate of perioperative seizures. The chosen cut-off point of 14 days for the inclusion of seizures as "perioperative" reflects our own practice standards. Patients are 
TABLE 3. Adjusted odds ratio for 90 -day readmission, $95 \%$ confidence intervals, and $p$ values for 342 patients undergoing first-time glioma resection

\begin{tabular}{|c|c|c|c|c|}
\hline \multirow[b]{2}{*}{ Covariates } & \multicolumn{2}{|c|}{ Univariate Analysis } & \multicolumn{2}{|c|}{ Multivariate Analysis } \\
\hline & OR $(95 \% \mathrm{Cl})$ & $p$ Value & OR $(95 \% \mathrm{Cl})$ & p Value \\
\hline \multicolumn{5}{|l|}{ Preoperative KPS score (ref: <70) } \\
\hline KPS score $\geq 70$ & $0.89(0.46-1.70)$ & 0.712 & & NA \\
\hline \multicolumn{5}{|l|}{ Tumor grade (ref: Grade I/II) } \\
\hline Grade III/IV & $1.26(0.69-2.31)$ & 0.460 & & NA \\
\hline EOR (nominal: STR, NTR, GTR) & & 0.080 & & NS \\
\hline \multicolumn{5}{|c|}{ Postoperative AED administration (ref: no AED) } \\
\hline AED & $0.62(0.29-1.32)$ & 0.210 & & NA \\
\hline \multicolumn{5}{|c|}{ Postoperative seizure (ref: no seizure) } \\
\hline Seizure & $4.45(1.69-11.7)$ & 0.001 & $3.65(1.36-9.79)$ & 0.010 \\
\hline \multicolumn{5}{|c|}{ Discharge disposition (ref: not home) ${ }^{*}$} \\
\hline Home & $0.54(0.31-0.97)$ & 0.036 & & NS \\
\hline \multicolumn{5}{|l|}{ XRT (Grade III/VI) (ref: no XRT) } \\
\hline XRT & $0.98(0.54-1.80)$ & 0.955 & & NA \\
\hline
\end{tabular}

routinely evaluated in our clinic for wound checks at 2 weeks postoperatively, during which patients are asked about any relevant postsurgical concerns. Additionally, an informal survey of recent practices suggested the clustering of seizures within this 2-week period. This survey was confirmed after our formal analysis, which indicated that the vast majority of postoperative seizures occurred within the first 13 days, after which seizure incidence dramatically diminished.

Indeed, the biological and clinical factors that predispose to the development of postoperative seizures are uncertain. Nothing in our data collection and analysis points to a modifiable factor that could be screened preoperatively to reduce seizures. Patients in both groups were similar in age, performance status, tumor cell type, and histological grade, as well as surgical approach and goal. There was a lower seizure rate for patients in whom GTR was achieved $(1.0 \%)$ than if NTR or STR was performed (7.1\%). Similarly, Chang et al. found that patients were 4 times less likely to experience postoperative seizures if GTR is achieved. ${ }^{3}$ This may not be surprising when considering that peritumoral cortical irritation and the disruption of inhibitory cellular networks are thought to contribute to elevated seizure risk in glioma patients. ${ }^{19,45,46}$ Incomplete seizure risk elimination would be expected to accompany the incomplete removal of the offending neoplasm.

Nevertheless, both study groups had similar rates of preoperative seizures and were similarly administered prophylactic AEDs for similar durations. Perhaps a certain (albeit modest) proportion of postoperative seizures may be unavoidable, and therefore imparting penalties on hospitals and physicians for these events seems unjust, if not misguided.

In 2000, the American Academy of Neurology published guidelines recommending against routine seizure prophylaxis in patients diagnosed with a brain tumor. ${ }^{15}$
In fact, there exist numerous reports advocating for the use of prophylactic antiseizure medications, ${ }^{21,31,41,42,48}$ and many others arguing against their utility. ${ }^{15,16,24,25,39,43}$ Despite conflicting evidence, the majority of neurosurgeons report administering anticonvulsants as prophylaxis. $.^{38} \mathrm{Re}-$ gardless, an examination of the efficacy or value of perioperative antiseizure medication is beyond the scope of this study and not among our objectives. Rather, our aim is to objectively quantify the clinical and economic impact of perioperative seizures in an effort to draw attention to its importance, particularly in light of looming reimbursement reforms.

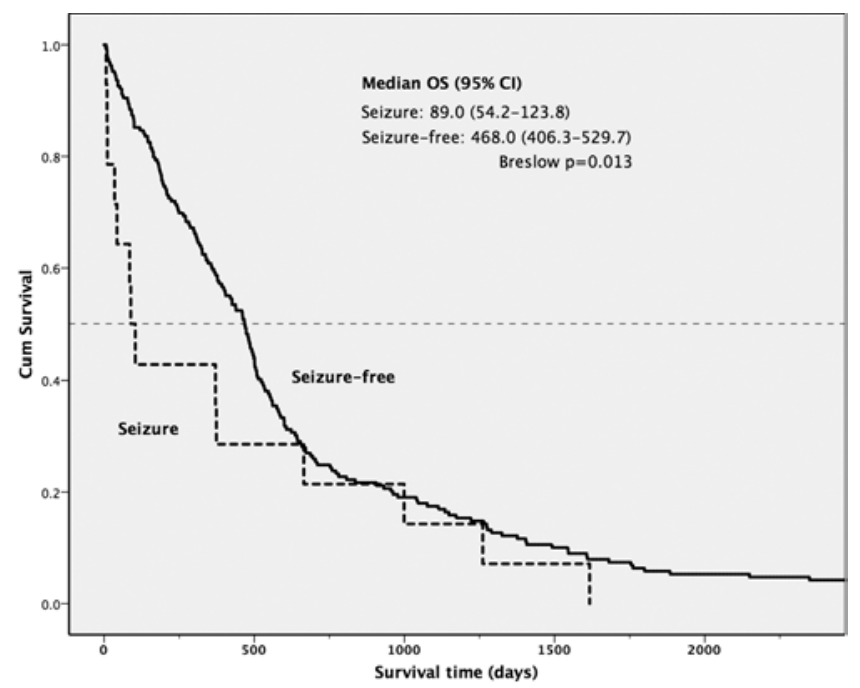

FIG. 2. Overall survival (OS) of 335 patients following first-time glioma resection. Kaplan-Meier estimates for the overall survival times (in days) for seizure (dashed line) and seizure-free (solid line) patients. The horizontal dashed line represents the $50 \%$ cumulative (cum) survival intercept. 


\section{Prolonged Hospital Stay and Discharge Disposition}

The dramatic prolongation of hospital stay-from an average of 3.6 to 6.8 days - can be partially explained by observing clinical decisions made in the wake of a postoperative seizure. While the ictal event may last only seconds or minutes, the cascade of events that transpires is one with which any experienced tumor surgeon has become familiar. After addressing the event itself with AED administration, neuroimaging is typically obtained to rule out hemorrhage, infarction, edema, or other structural complications. Next, a formal neurology consultation is frequently sought, which is often accompanied by EEG evaluation. In our cohort, half of the patients with a postoperative seizure received a neurology consultation, and the vast majority of these patients underwent both EEG and imaging evaluation. Regardless of the outcome of these studies, AEDs are generally added and patients are kept in the ICU longer to undergo continued frequent neurological examinations. A 2-day postoperative stay can quickly turn into a week upon the conclusion of the additional testing and observation period-even if (and more often when) clinically appreciable postictal deficits or complications are absent.

The occurrence of perioperative seizures was not associated with the discharge destination $(p=0.068)$, although patients discharged to home were less likely to be readmitted within 90 days $(\mathrm{p}=0.036)$. The direct and indirect costs related to increased LOS and discharging patients to a rehabilitation facility were not considered in this analysis, although such expenditures could be expected to increase the economic burden of perioperative seizures.

\section{Hospital Readmissions}

The psychological consequences of a perioperative seizure are not limited to the index hospitalization and are certainly not limited to the surgeon. The negative impact of brain tumor-related epilepsy on quality of life has been well described ${ }^{6,9,22,30}$ and is reflected in the data presented here. Patients and families discharged following a perioperative seizure are discharged harboring a sense of anticipation of the next seizure. This heightened anxiety may explain the more frequent ED presentations and ultimately higher rates of readmission for neurological reasons. Patients in our seizure cohort presented to the ED more than twice as often as those without perioperative seizures ( $44.4 \%$ vs $19.0 \%$ ). Interestingly, the majority of these patients presented for neurological reasons not deemed to be seizures.

Overall, the primary reasons for readmission were for neurological (35\%) and infectious morbidity (16.3\%), corroborating previously published data. ${ }^{5,33}$ Dickinson et al. ${ }^{5}$ found that neurological symptoms accounted for more than a third of readmissions (37.0\%) in 362 patients with glioblastoma, followed by infectious complications (29.6\%). In a separate review of 2774 patients with high-grade lesions, $15.8 \%$ of patients were readmitted within 30 days of surgery. While our overall readmission rate was higher $(20 \%)$ at 90 days, our window of inclusion was 3 times greater. This longer period of follow-up was chosen to reflect changes due to both internal institutional reviewers as well as external reimbursement agencies that have begun extending the postoperative complication window from
30 to 90 days. ${ }^{2,37}$ Indeed, recent data from large, prospective trials published in the cardiac literature (whence the 30-day benchmark was initially established) indicates that only a fraction $(23 \%)$ of treatment-related readmissions occur within 30 days, whereas $19 \%$ occur between 31 and 60 days and an astonishing $58 \%$ occur after 60 days. ${ }^{14,34}$

In addition to postoperative seizures, a patient's discharge disposition was closely associated with the rate of 90-day readmission. Specifically, patients discharged home were half as likely to be readmitted (OR 0.54) (Table 3). Similarly, in a glioblastoma population, Dickinson et al. found that patients discharged to a rehabilitation or transfer facility were approximately 4 times as likely to require readmission. ${ }^{5}$ Unlike in their population, we did not find that the preoperative KPS score influenced the odds of readmission $(\mathrm{p}=0.712)$.

\section{Survival}

While several studies examine the association between seizures and quality of life in glioma patients, $, 10,22,44$ this study is the first to specifically analyze the relationship between perioperative seizures and overall survival. In our cohort, patients who suffered an immediate postoperative seizure had a median overall survival period of 3 months in comparison with 15.6 months in seizure-free patients. Woo et al. reported the decreased survival of nearly 6 months in patients who experienced seizures anytime during their disease state, though this trend did not reach statistical significance $(\mathrm{p}=0.064){ }^{47}$ Many studies associate preoperative seizures with improved overall survival, but because lower grade lesions have epileptogenic predilection, many conclude that the survival advantage is simply because seizures serve as a proxy for low-grade histology. ${ }^{26,27,40}$ Contrary to preoperative seizures, here we show that postoperative seizures, specifically those within 14 days of surgery, carry a poorer prognosis. The influence of perioperative seizures on overall survival remains after adjusting for tumor grade and EOR (which themselves are strong, independent predictors of survival). In summary, our analysis demonstrates that the development of perioperative seizure was independently and inversely related to overall survival, particularly within the 1 st year after index surgery. Future studies that incorporate other glioma variables, including tumor-specific markers and mutations in larger study populations, may help uncover the reasons for the observed survival disadvantage.

\section{Limitations}

This study has several limitations, some of which are inherent to the retrospective exploration of hospital data. In a minority of patients early in the series, there was limited electronic medical documentation of some radiographic and outpatient follow-up details. Nonetheless, all included patients had sufficient records to allow reliable analysis of seizure and survival data. Next, because we included seizures up to 14 days postoperatively, many seizures were not captured in the hospital. Conservative criteria for seizure diagnosis in the outpatient setting may have translated into the underdiagnosis - and therefore underreporting-of seizures. Additionally, the readmission data may not be representative of the true rate of hospital 
visits if the patients were evaluated at an outside facility and we did not become aware of such a visit. Nuño et al. found that as much as $37 \%$ of patients were readmitted to the nonindex hospital. ${ }^{33}$ Our review of follow-up visits with both surgeons and oncologists captured many such external admissions, though conceivably some may have been missed. Finally, our findings did not include an analysis of glioma biomarkers and their potential influence on perioperative epileptogenicity. It will be important for future studies to incorporate such characteristics, which are known to have strong diagnostic and prognostic value.

\section{Conclusions}

Perioperative seizures following glioma resection occur in a meaningful proportion of patients and result in prolonged hospitalization, increased readmissions, and shorter overall survival. In the setting of health care reforms that penalize providers for surgical morbidity, efforts to identify markers for the development of seizures and to further mitigate perioperative seizures after glioma surgery should remain a priority among neurosurgeons, neurocritical care physicians, and neurologists alike.

\section{References}

1. Bähr O, Hermisson M, Rona S, Rieger J, Nussbaum S, Körtvelyessy $\mathrm{P}$, et al: Intravenous and oral levetiracetam in patients with a suspected primary brain tumor and symptomatic seizures undergoing neurosurgery: the HELLO trial. Acta Neurochir (Wien) 154:229-235, 2012

2. Brooke BS, Goodney PP, Kraiss LW, Gottlieb DJ, Samore MH, Finlayson SRG: Readmission destination and risk of mortality after major surgery: an observational cohort study. Lancet 386:884-895, 2015

3. Chang EF, Potts MB, Keles GE, Lamborn KR, Chang SM, Barbaro NM, et al: Seizure characteristics and control following resection in 332 patients with low-grade gliomas. J Neurosurg 108:227-235, 2008

4. Deutschman CS, Haines SJ: Anticonvulsant prophylaxis in neurological surgery. Neurosurgery 17:510-517, 1985

5. Dickinson H, Carico C, Nuño M, Mukherjee D, Ortega A, Black KL, et al: Unplanned readmissions and survival following brain tumor surgery. J Neurosurg 122:61-68, 2015

6. Dinapoli L, Maschio M, Jandolo B, Fabi A, Pace A, Sperati F, et al: Quality of life and seizure control in patients with brain tumor-related epilepsy treated with levetiracetam monotherapy: preliminary data of an open-label study. Neurol Sci 30:353-359, 2009

7. Dobson A, El-Gamil A, Heath S, Berger G, DaVanzo JE: Working Paper \#4: Baseline Statistics of Acute Care Hospital Readmissions by Episode Type for Select MSDRGs and Chronic Conditions. Clinically Appropriate and Cost-Effective Placement (CACEP) Project Working Paper Series. Vienna, VA: Dobson DaVanzo \& Associates, 2012

8. Drappatz J, Schiff D, Kesari S, Norden AD, Wen PY: Medical management of brain tumor patients. Neurol Clin 25:1035-1071, ix, 2007

9. Englot DJ, Berger MS, Barbaro NM, Chang EF: Predictors of seizure freedom after resection of supratentorial low-grade gliomas. A review. J Neurosurg 115:240-244, 2011

10. Englot DJ, Berger MS, Chang EF, Garcia PA: Characteristics and treatment of seizures in patients with high-grade glioma: a review. Neurosurg Clin N Am 23:227-235, vii-viii, 2012

11. Epstein AM, Jha AK, Orav EJ: The relationship between hospital admission rates and rehospitalizations. N Engl J Med 365:2287-2295, 2011
12. Forsyth PA, Weaver S, Fulton D, Brasher PMA, Sutherland G, Stewart D, et al: Prophylactic anticonvulsants in patients with brain tumour. Can J Neurol Sci 30:106-112, 2003

13. Foy PM, Copeland GP, Shaw MD: The natural history of postoperative seizures. Acta Neurochir (Wien) 57:15-22, 1981

14. Gheorghiade M, Pang PS, Ambrosy AP, Lan G, Schmidt P, Filippatos G, et al: A comprehensive, longitudinal description of the in-hospital and post-discharge clinical, laboratory, and neurohormonal course of patients with heart failure who die or are re-hospitalized within 90 days: analysis from the EVEREST trial. Heart Fail Rev 17:485-509, 2012

15. Glantz MJ, Cole BF, Forsyth PA, Recht LD, Wen PY, Chamberlain MC, et al: Practice parameter: anticonvulsant prophylaxis in patients with newly diagnosed brain tumors. Report of the Quality Standards Subcommittee of the American Academy of Neurology. Neurology 54:1886-1893, 2000

16. Glantz MJ, Cole BF, Friedberg MH, Lathi E, Choy H, Furie $\mathrm{K}$, et al: A randomized, blinded, placebo-controlled trial of divalproex sodium prophylaxis in adults with newly diagnosed brain tumors. Neurology 46:985-991, 1996

17. Harris PA, Taylor R, Thielke R, Payne J, Gonzalez N, Conde JG: Research electronic data capture (REDCap) - a metadata-driven methodology and workflow process for providing translational research informatics support. J Biomed Inform 42:377-381, 2009

18. Hernandez AF, Greiner MA, Fonarow GC, Hammill BG, Heidenreich PA, Yancy CW, et al: Relationship between early physician follow-up and 30-day readmission among Medicare beneficiaries hospitalized for heart failure. JAMA 303:1716-1722, 2010

19. James E, Varelas PN: Brain tumor and seizures: incidence, pathophysiology, diagnosis and treatment, in Garami M (ed): Management of CNS Tumors. 2011, pp 1-15

20. Jencks SF, Williams MV, Coleman EA: Rehospitalizations among patients in the Medicare fee-for-service program. $\mathbf{N}$ Engl J Med 360:1418-1428, 2009

21. Kern K, Schebesch KM, Schlaier J, Hansen E, Feigl GC, Brawanski AT, et al: Levetiracetam compared to phenytoin for the prevention of postoperative seizures after craniotomy for intracranial tumours in patients without epilepsy. J Clin Neurosci 19:99-100, 2012

22. Klein M, Engelberts NHJ, van der Ploeg HM, KasteleijnNolst Trenité DGA, Aaronson NK, Taphoorn MJB, et al: Epilepsy in low-grade gliomas: the impact on cognitive function and quality of life. Ann Neurol 54:514-520, 2003

23. Klimek M, Dammers R: Antiepileptic drug therapy in the perioperative course of neurosurgical patients. Curr Opin Anaesthesiol 23:564-567, 2010

24. Komotar RJ, Raper DMS, Starke RM, Iorgulescu JB, Gutin $\mathrm{PH}$ : Prophylactic antiepileptic drug therapy in patients undergoing supratentorial meningioma resection: a systematic analysis of efficacy. J Neurosurg 115:483-490, 2011

25. Kuijlen JM, Teernstra OP, Kessels AG, Herpers MJ, Beuls EA: Effectiveness of antiepileptic prophylaxis used with supratentorial craniotomies: a meta-analysis. Seizure 5:291298, 1996

26. Leighton C, Fisher B, Bauman G, Depiero S, Stitt L, MacDonald D, et al: Supratentorial low-grade glioma in adults: an analysis of prognostic factors and timing of radiation. J Clin Oncol 15:1294-1301, 1997

27. Lote K, Stenwig AE, Skullerud K, Hirschberg H: Prevalence and prognostic significance of epilepsy in patients with gliomas. Eur J Cancer 34:98-102, 1998

28. Manaka S, Ishijima B, Mayanagi Y: Postoperative seizures: epidemiology, pathology, and prophylaxis. Neurol Med Chir (Tokyo) 43:589-600, 2003

29. Marcus LP, McCutcheon BA, Noorbakhsh A, Parina RP, Gonda DD, Chen C, et al: Incidence and predictors of 30-day 
readmission for patients discharged home after craniotomy for malignant supratentorial tumors in California (1995-

2010). J Neurosurg 120:1201-1211, 2014

30. Maschio M, Dinapoli L, Sperati F, Pace A, Fabi A, Vidiri A, et al: Levetiracetam monotherapy in patients with brain tumor-related epilepsy: seizure control, safety, and quality of life. J Neurooncol 104:205-214, 2011

31. Merrell RT, Anderson SK, Meyer FB, Lachance DH: Seizures in patients with glioma treated with phenytoin and levetiracetam. J Neurosurg 113:1176-1181, 2010

32. North JB, Penhall RK, Hanieh A, Frewin DB, Taylor WB: Phenytoin and postoperative epilepsy. A double-blind study. J Neurosurg 58:672-677, 1983

33. Nuño M, Ly D, Ortega A, Sarmiento JM, Mukherjee D, Black KL, et al: Does 30-day readmission affect long-term outcome among glioblastoma patients? Neurosurgery 74:196-205, 2014

34. O'Connor CM, Miller AB, Blair JEA, Konstam MA, Wedge $\mathrm{P}$, Bahit MC, et al: Causes of death and rehospitalization in patients hospitalized with worsening heart failure and reduced left ventricular ejection fraction: results from Efficacy of Vasopressin Antagonism in Heart Failure Outcome Study with Tolvaptan (EVEREST) program. Am Heart J 159:8418449, 849.e1, 2010

35. Patient Protection and Affordable Care Act of 2010, Pub Law 111-148, 124 Stat. 127, Sec. 6301 (March 23, 2010) (http:// www.gpo.gov/fdsys/pkg/PLAW-111publ148/pdf/PLAW111publ148.pdf)

36. Rosenbaum S: The Patient Protection and Affordable Care Act: implications for public health policy and practice. Public Health Rep 126:130-135, 2011

37. Sadot E, Brennan MF, Lee SY, Allen PJ, Gönen M, Groeger JS, et al: Readmission after pancreatic resection: causes and causality pattern. Ann Surg Oncol 21:4342-4350, 2014

38. Siomin V, Angelov L, Li L, Vogelbaum MA: Results of a survey of neurosurgical practice patterns regarding the prophylactic use of anti-epilepsy drugs in patients with brain tumors. J Neurooncol 74:211-215, 2005

39. Sirven JI, Wingerchuk DM, Drazkowski JF, Lyons MK, Zimmerman RS: Seizure prophylaxis in patients with brain tumors: a meta-analysis. Mayo Clin Proc 79:1489-1494, 2004

40. Stark AM, van de Bergh J, Hedderich J, Mehdorn HM, Nabavi A: Glioblastoma: clinical characteristics, prognostic factors and survival in 492 patients. Clin Neurol Neurosurg 114:840-845, 2012

41. Temkin NR: Antiepileptogenesis and seizure prevention trials with antiepileptic drugs: meta-analysis of controlled trials. Epilepsia 42:515-524, 2001

42. Temkin NR, Dikmen SS, Wilensky AJ, Keihm J, Chabal S, Winn HR: A randomized, double-blind study of phenytoin for the prevention of post-traumatic seizures. N Engl J Med 323:497-502, 1990
43. Tremont-Lukats IW, Ratilal BO, Armstrong T, Gilbert MR: Antiepileptic drugs for preventing seizures in people with brain tumors. Cochrane Database Syst Rev (2):CD004424, 2008

44. van Breemen MSM, Wilms EB, Vecht CJ: Epilepsy in patients with brain tumours: epidemiology, mechanisms, and management. Lancet Neurol 6:421-430, 2007

45. Vecht CJ, van Breemen M: Optimizing therapy of seizures in patients with brain tumors. Neurology 67 (12 Suppl 4):S10S13, 2006

46. Wen PY, Marks PW: Medical management of patients with brain tumors. Curr Opin Oncol 14:299-307, 2002

47. Woo PYM, Chan DTM, Chan KY, Wong WK, Po YC, Kwok JCK, et al: Risk factors for seizures and antiepileptic drugassociated adverse effects in high-grade glioma patients: A multicentre, retrospective study in Hong Kong. Surg Pract 19:2-8, 2015

48. Zachenhofer I, Donat M, Oberndorfer S, Roessler K: Perioperative levetiracetam for prevention of seizures in supratentorial brain tumor surgery. J Neurooncol 101:101-106, 2011

\section{Disclosures}

The authors report no conflict of interest concerning the materials or methods used in this study or the findings specified in this paper.

\section{Author Contributions}

Conception and design: Dewan, Chambless. Acquisition of data: White-Dzuro, Brinson. Analysis and interpretation of data: Dewan. Drafting the article: Dewan, White-Dzuro. Critically revising the article: Dewan, White-Dzuro, Brinson, Thompson. Reviewed submitted version of manuscript: all authors. Approved the final version of the manuscript on behalf of all authors: Dewan. Statistical analysis: Dewan. Administrative/technical/ material support: Brinson, Chambless. Study supervision: Thompson, Chambless.

\section{Supplemental Information \\ Previous Presentations}

Portions of this work were presented at the Congress of Neurological Surgeons Annual Meeting, New Orleans, Louisiana, October 2015.

\section{Correspondence}

Michael C. Dewan, Department of Neurological Surgery, Vanderbilt Medical Center, T-4224 Medical Center N, Nashville, TN 37232-2380. email: michael.dewan@vanderbilt.edu. 